\title{
October 2015 Imaging Case of the Month
}

\author{
Philip Ho, MD \\ Stacey Black, MD \\ Clinton Jokerst, MD
}

Department of Medical Imaging

Banner University Medical Center

Tucson, AZ

Clinical History: A 68-year old Hispanic man presented to the emergency department with dry cough for two days and was found to be hypoxic, with $\mathrm{O}_{2}$ saturation in the high 80 's. The patient's clinical history is significant for remote 3 year smoking history and former occupation as a miner. Frontal and lateral chest radiography (Figure 1) was obtained.

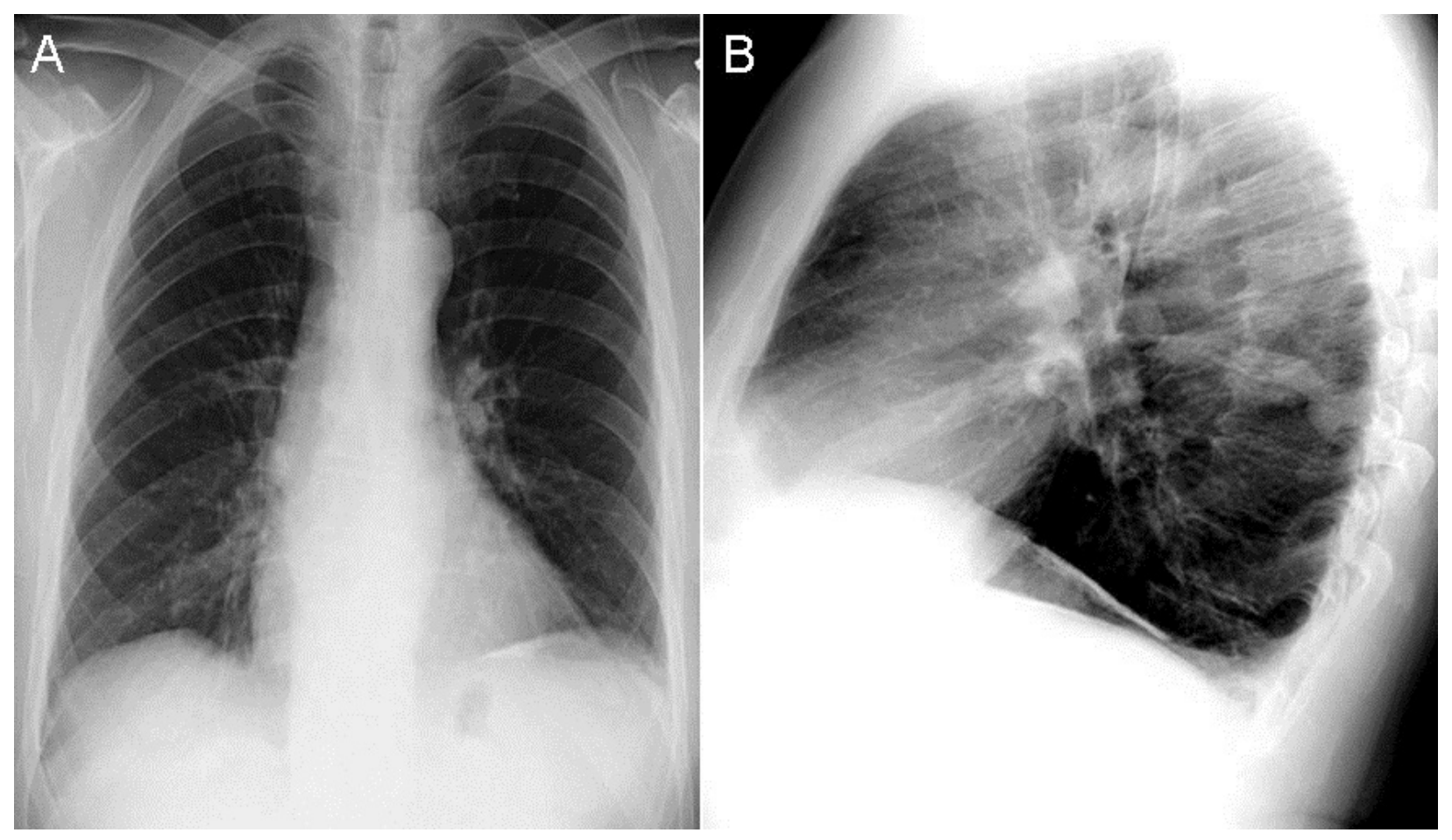

Figure 1. Frontal (panel A) and lateral (panel B) chest radiograph.

Based on the appearance of the chest radiograph, which of the following is the least likely diagnosis?

1. Osteophyte

2. Peripheral nerve sheath tumor

3. Pneumonia

4. Pulmonary arteriovenous malformation (AVM) 


\section{Correct! \\ 1. Osteophyte}

There is a tubular opacity that appears to be connecting to the hilum on the lateral view (Figure 2).
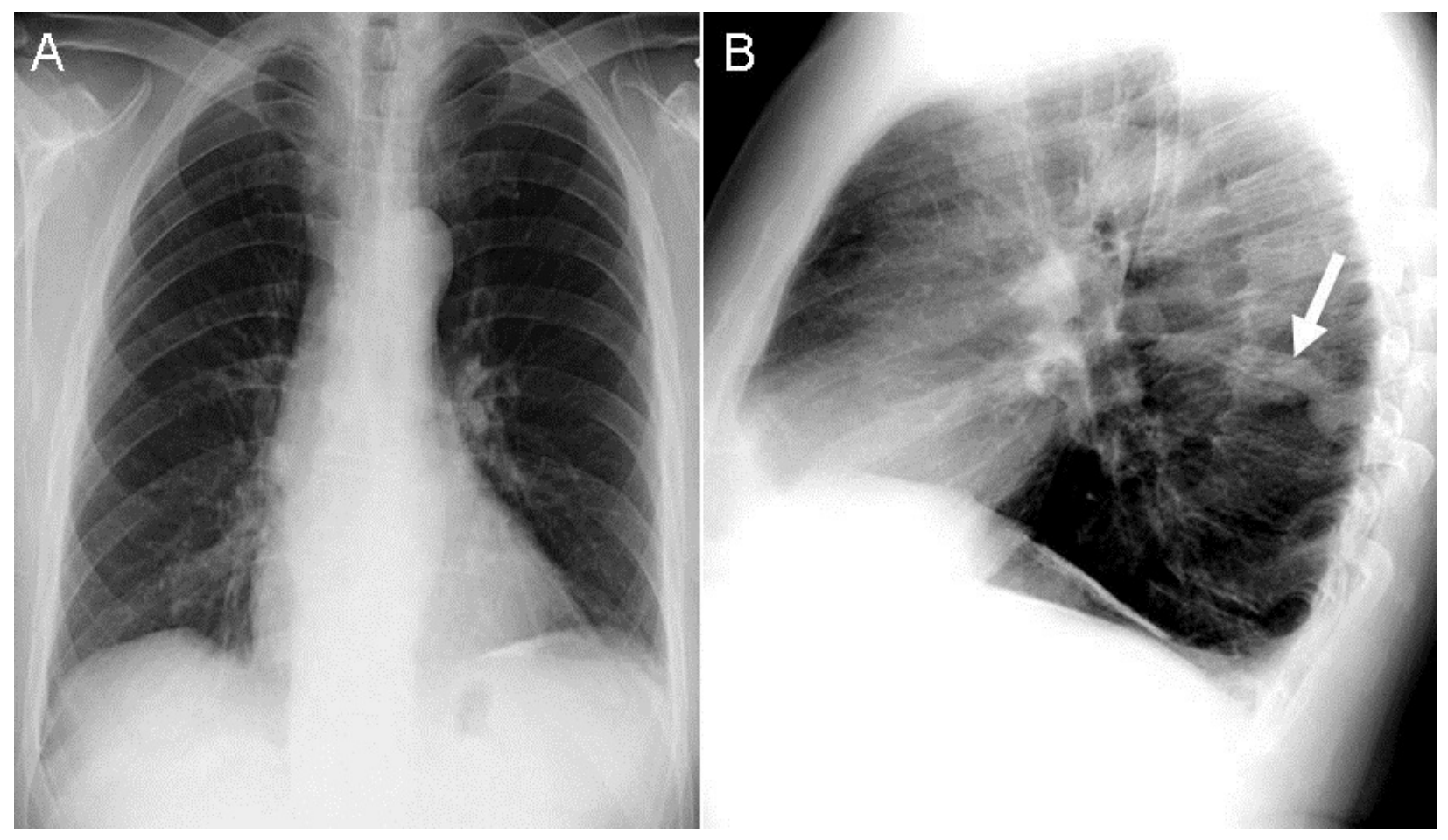

Figure 2. Frontal and lateral chest radiographs show that the left hilum is somewhat "dense" compared with the right hilum, caused by an abnormal opacity projecting over the spine (arrow) on the lateral view.

All the answer choices are potential possibilities; however the chest radiographic findings would be an unusual appearance for an osteophyte, especially as the patient does not have osteophyte formation elsewhere in the spine. Pneumonia is possible given the patient's history of cough. A pulmonary AVM and a posterior mediastinal mass such as a peripheral nerve sheath tumor are possible. Note there is partial calcification of the left hemidiaphragm, consistent with asbestos-related pleural disease.

Contrast-enhanced thoracic CT (Figure 3) was performed for further evaluation of the chest radiographic findings. 


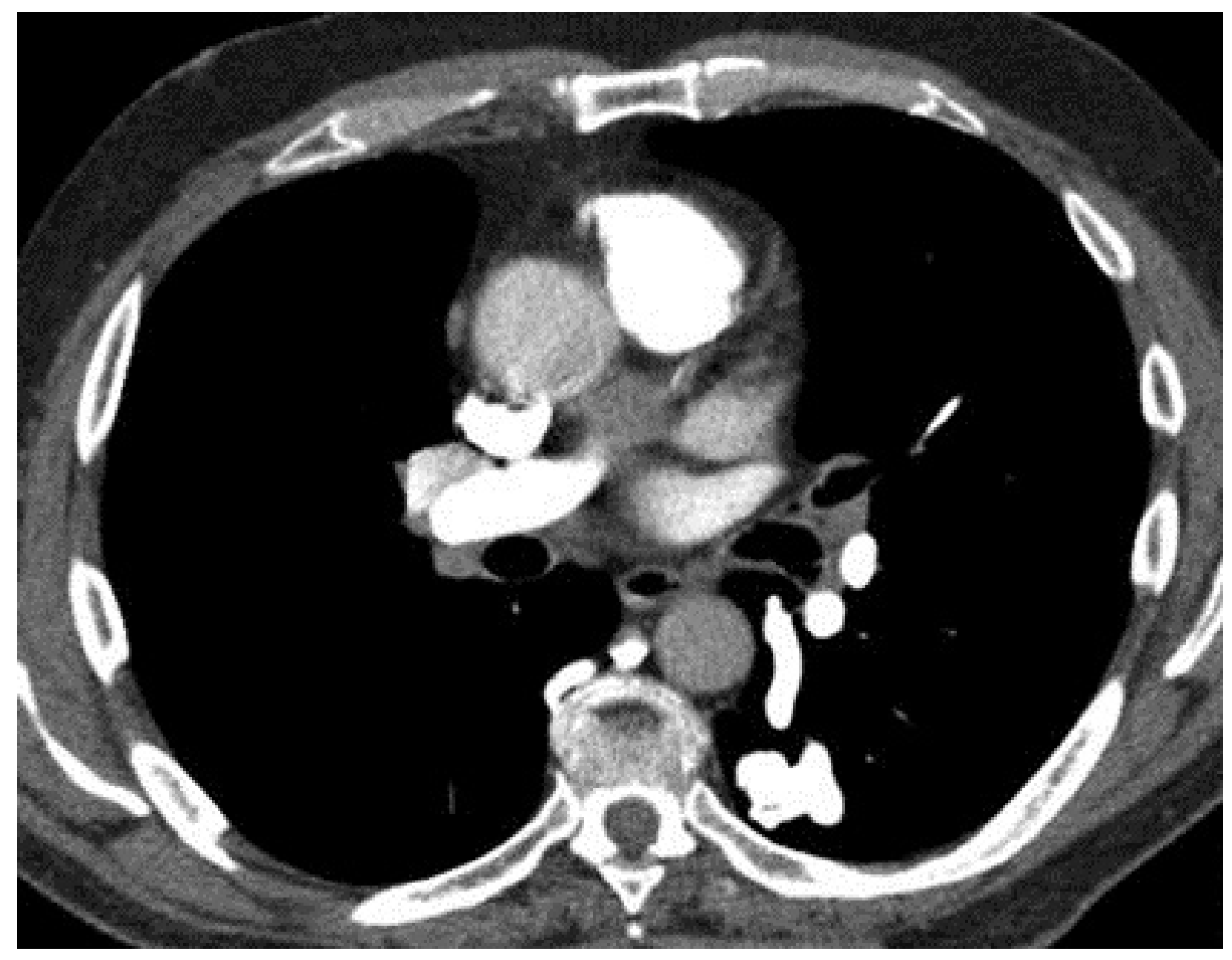

Figure 3. Enhanced thoracic CT displayed in soft tissue windows.

Based on the thoracic CT appearance, what is the diagnosis?

1. Bacterial pneumonia

2. Bronchogenic malignancy

3. Pleural plaque

4. Pulmonary AVM 


\section{Correct! \\ 4. Pulmonary AVM}

Within the posterior left lung adjacent to the spine there is a vascular lesion that has a feeding artery and a draining vein, compatible with an AVM. None of the other answers explain these findings. Contrast enhanced CT demonstrates that this abnormality is a vascular lesion, and not a nodule/mass. Biopsy of such a lesion could result in catastrophic bleeding. Administration of intravenous contrast can be very helpful when evaluating a pulmonary lesion which could be vascular in nature.

The patient was referred to interventional radiology for embolization of the pulmonary AVM.

What is the embolization agent of choice used for AVM embolotherapy?
1. Coils
2. Gel Foam
3. Glue
4. Particles 


\section{Correct!}

\section{Coils}

Pulmonary AVMs create an intrapulmonary right-to-left shunt, which can predispose patients to hypoxia (as in the case of this patient) and paradoxical systemic embolization, which can lead to complications such as stroke and brain abscess. Coils are the agent of choice for embolotherapy. Amplatzer vascular plugs have also been used to treat very large AVMs. The remaining answer choices are embolization agents that travel distally and would lead to systemic embolization and devastating consequences related to non-target embolization. Classically, feeding arteries $3 \mathrm{~mm}$ or larger in diameter are selectively embolized and the initial coil is oversized approximately $20 \%$ to minimize the risk of the coil passing through the AVM. For this patient, there were multiple tortuous feeding arteries feeding the pulmonary $A V M$, as seen in a representative pulmonary angiogram (Figure 4).

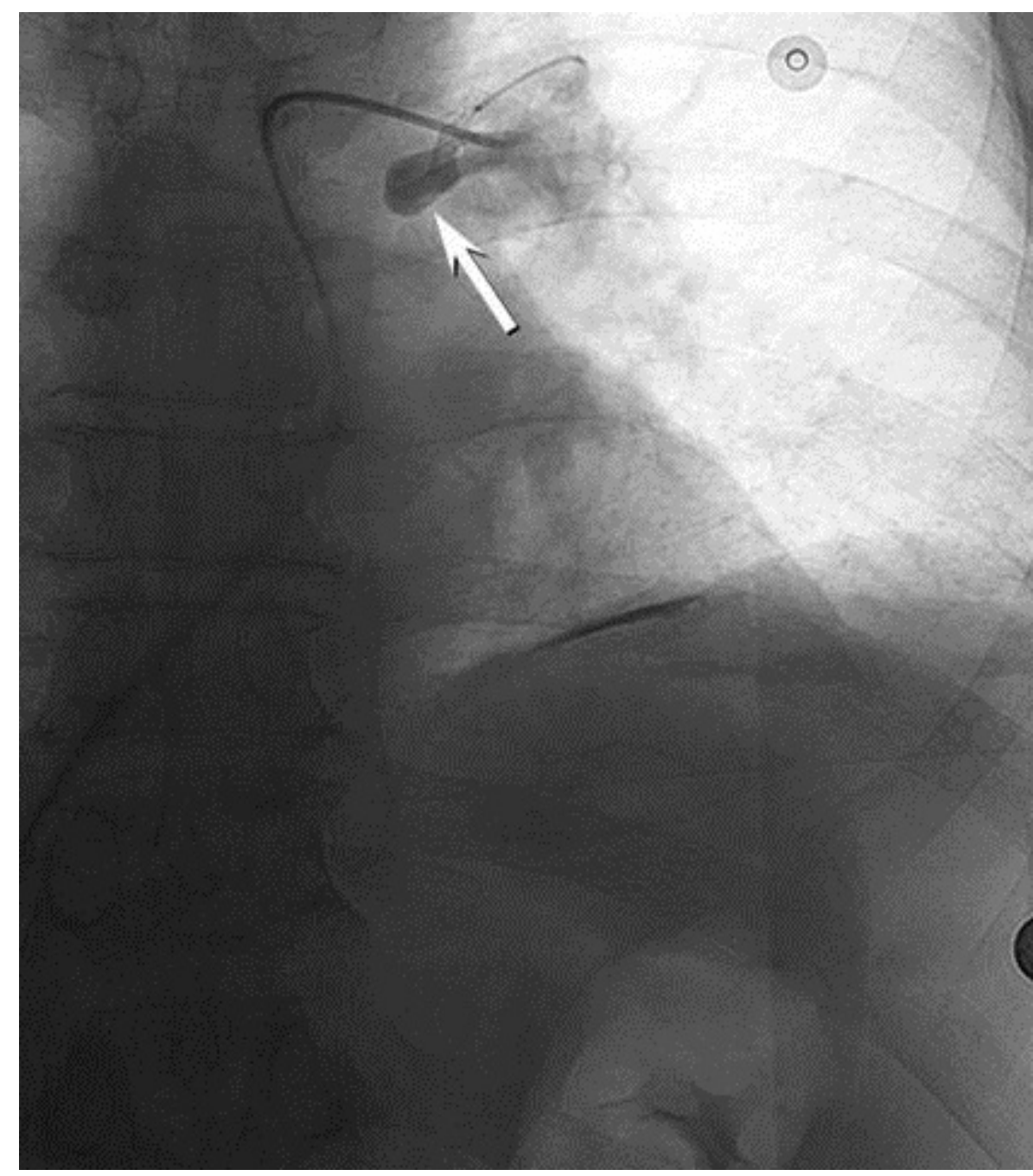

Figure 4. Catheter pulmonary angiography performed following selective injection of the left pulmonary arterial system shows opacification of the pulmonary arteriovenous malformation (arrow). 
Post procedure chest radiographs (Figure 5) show that multiple coils were deployed for adequate treatment.

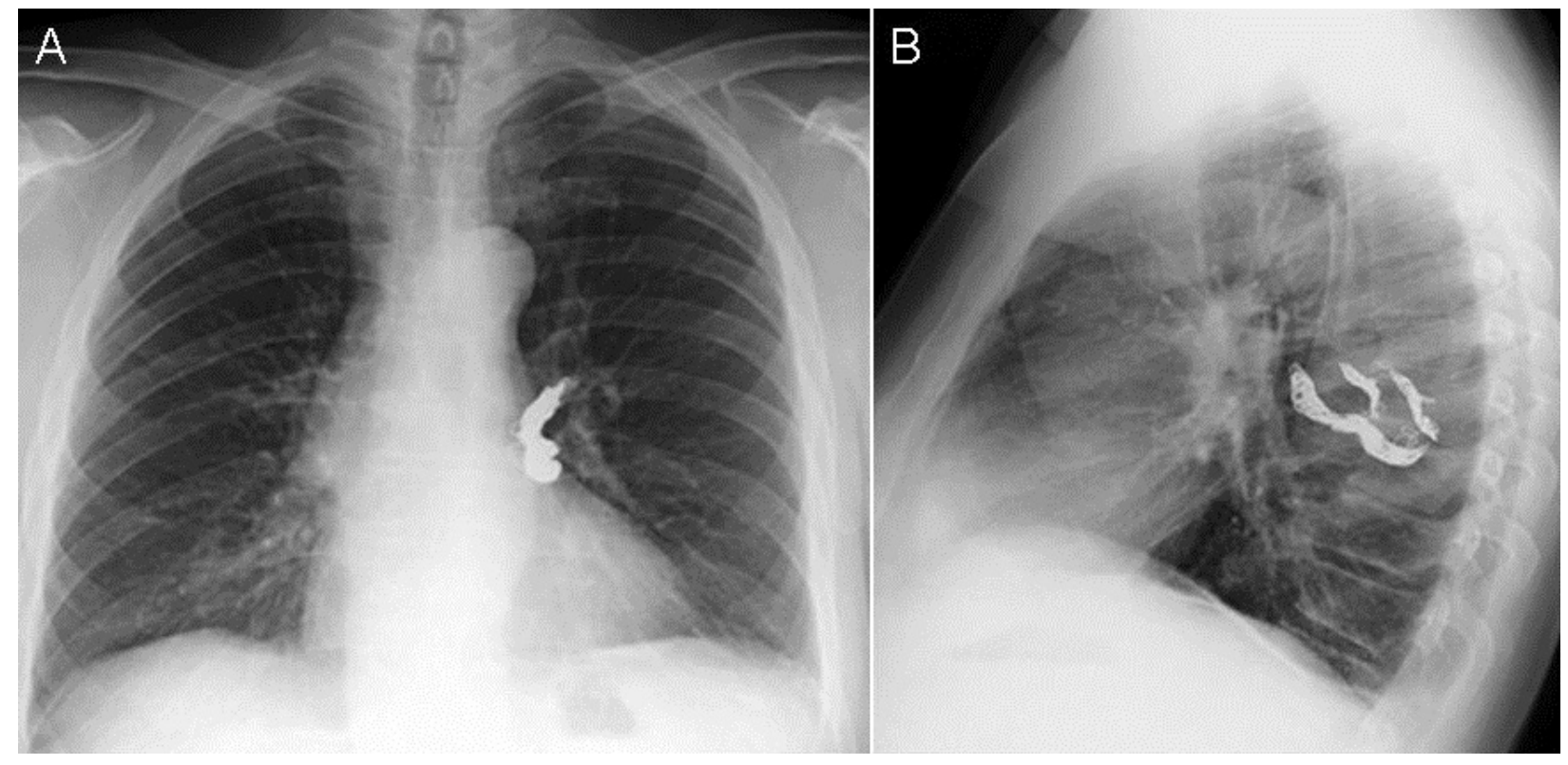

Figure 5. Frontal (A) and lateral (B) chest radiograph following embolotherapy for the left-sided pulmonary arteriovenous malformation shows endovascular placement of multiple metallic coils.

Patients typically receive a CTA at 1-3 months following embolization therapy, then at 612 months, and then every 3 years unless otherwise required. Treatment is considered successful at CTA when the draining vein decreases in size and the AVM nidus involutes. If this does not occur, then repeat angiography must be performed as this could indicate recanalization or incomplete embolization.

The patient has history of epistaxis, raising concern for hereditary hemorrhagic telangiectasia $(\mathrm{HHT})$.

Which of the following is not part of the Curacao criteria (2000) for diagnosing HHT?
1. AVMs
2. Epistaxis
3. Family History
4. Stroke
5. Telangiectasia 


\section{Correct!}

\section{Stroke}

Strokes are potential complication of AVMs but are not part of the criteria for diagnosing hereditary hemorrhagic telangiectasia (formerly known as Osler-Weber-Rendu Syndrome). Epistaxis is spontaneous and recurrent in the setting of HHT. Telangiectasias are usually multiple and are seen on the nose, lips, oral mucosa, and fingers tips. Pulmonary AVMs are not invariably present in patients with HHT, and they can also be present systemically, such as the liver, spine, and brain. Family history in the context of HHT refers to a first degree relative. These are the 4 criteria for diagnosing HHT, with "definite HHT" defined as three or more criteria present, "possible or suspected HHT" defined as two criteria present, and "HHT unlikely" if no or only 1 criterion is present. These clinical features have been deemed useful for discriminating affected from non-affected older adults and establishing the diagnosis in younger adults and children. More recently, HHT can be diagnosed with genetic testing, which has been useful in children and young patients that do not yet have the typical clinical manifestations.

Diagnosis: pulmonary AVM

\section{References}

1. Cartin-Ceba R, Swanson KL, Krowka MJ. Pulmonary arteriovenous malformations. Chest. 2013;144:1033-44. [CrossRef] [PubMed]

2. Faughnan ME, Palda VA, Garcia-Tsao G, et al. International guidelines for the diagnosis and management of hereditary hemorrhagic telangiectasia. J Med Genet 2011;48:73-87. [CrossRef] [PubMed]

3. Meek ME, Meek JC, Beheshti MV. Management of pulmonary arteriovenous malformations. Semin Interv Radiol 2011;28:24-31. [CrossRef] [PubMed] 